University of Wollongong

Research Online

Faculty of Engineering - Papers (Archive)

Faculty of Engineering and Information

Sciences

February 2003

\title{
Induced charge-density oscillation under a quantizing magnetic field and intense terahertz radiation
}

M. Fujita

Tokai University, Japan

T. Toyoda

Tokai University, Japan

J. C. Cao

Chinese Academy of Sciences, Shanghai, China

C. Zhang

University of Wollongong, czhang@uow.edu.au

Follow this and additional works at: https://ro.uow.edu.au/engpapers

Part of the Engineering Commons

https://ro.uow.edu.au/engpapers/235

\section{Recommended Citation}

Fujita, M.; Toyoda, T.; Cao, J. C.; and Zhang, C.: Induced charge-density oscillation under a quantizing magnetic field and intense terahertz radiation 2003.

https://ro.uow.edu.au/engpapers/235

Research Online is the open access institutional repository for the University of Wollongong. For further information contact the UOW Library: research-pubs@uow.edu.au 


\title{
Induced charge-density oscillation under a quantizing magnetic field and intense terahertz radiation
}

\author{
M. Fujita, ${ }^{1}$ T. Toyoda, ${ }^{1}$ J. C. Cao, ${ }^{2}$ and C. Zhang $^{3}$ \\ ${ }^{1}$ Department of Physics, Tokai University, Kitakaname 1117, Hiratsuka, Kanagawa 259-1292, Japan \\ ${ }^{2}$ Shanghia Institute of Microsystem and Information Technology, Chinese Academy of Sciences, Shanghai, China \\ ${ }^{3}$ Department of Physics and Institute of Superconducting \\ and Electronic Materials, University of Wollongong, New South Wales 2522, Australia \\ (Received 27 August 2002; revised manuscript received 15 October 2002; published 12 February 2003)
}

\begin{abstract}
The time-dependent Schrödinger equation for a two-dimensional electron gas in a strong magnetic field and an intense terahertz laser field is solved by constructing unitary transformations. We calculate the chargedensity fluctuation under a weak probing potential. It is found that the induced charge density oscillates rapidly in time with two characteristic frequencies, the cyclotron frequency and the laser frequency.
\end{abstract}

DOI: 10.1103/PhysRevB.67.075105

PACS number(s): 71.10.Ca, 71.70.Di

\section{INTRODUCTION}

Terahertz frequency resonantly matches with virtually all characteristic energies in low-dimensional semiconductors, such as quantum wells and quantum wires. Because of this energy resonance, terahertz radiation plays an important role in understanding the electronic and optical properties of lowdimensional semiconductor systems and many optoelectronic devices. Recently there has been a rapid development of tunable terahertz $(\mathrm{THz})$ laser sources based on semiconductor structures, ${ }^{1,2}$ and a widely tunable continuous-wave terahertz (THz) generation was achieved experimentally in strained Ge. ${ }^{3}$ These radiation sources can provide linearly polarized laser radiation in the terahertz regime. ${ }^{4-11} \mathrm{THz}$ lasers have been applied to an experimental investigation of nonlinear transport and optical properties in electron gases such as low-dimensional semiconductor systems. Many interesting terahertz phenomena have been investigated, including resonant absorption, ${ }^{4}$ the photon enhanced hot-electron effect, ${ }^{7}$ $\mathrm{THz}$ photon-induced impact ionization, ${ }^{8}$ the LO-phonon bottleneck effect, ${ }^{9} \mathrm{THz}$ photon assisted tunneling, ${ }^{10} \mathrm{THz}$ cyclotron resonance, ${ }^{11}$ and the Thz switching effect in resonant tunneling diodes. ${ }^{12-14}$

The problem of an electron subjected simultaneously to a quantizing magnetic field and an intense terahertz laser is of particular interest due to the interplay between terahertz photons and cyclotron orbits. In the previous work, ${ }^{15,16}$ we calculated the electronic states using unitary transformations for a free electron in a magnetic field. If the laser field is polarized in the $y$ direction and the Laudau gauge is used for the static magnetic field, these unitary transformations depend on $t, x$, and $p_{x}$.

In this work, we study the problem of electronic properties of a two-dimensional electron moving in a weak potential $V(x, y)$ under a quantizing magnetic field and an intense laser. We first construct the unitary transformations that eliminate the laser field. For a weak potential $V(x, y)$, the problem can be solved with two different approaches. In the first approach, one constructs the unitary transformations in the absence of $V(x, y)$ and obtains the exact wave function in the presence of the magnetic field and the laser field. One then use this time-dependent wave function as the base state to calculate the wave function to the lowest order in $V(x, y)$. In the second approach, one constructs unitary transformations in the presence of $V(x, y)$, such transformations will be dependent on all dynamical variables $t, x, p_{x}, y$, and $p_{y}$. After eliminating the laser field, the potential $V(x, y)$ becomes time dependent, $V[x(t), y(t)]$. One can then obtain the approximate wave function to the first order of this timedependent potential. These two approaches are equivalent for the single electron states. However, the statistical properties of many electron systems cannot be easily defined in the first approach because the base states are not formed by eigenfunctions. This problem can be avoided in the second approach. In this work we shall use the second approach to calculate the density fluctuation due to $V(x, y)$.

\section{UNITARY TRANSFORMATIONS}

We consider a two-dimensional electron gas under a uniform static magnetic field and an intense laser radiation. We choose the static magnetic field to be along the $z$ direction and the laser electric field to be along the $y$ direction, $\mathbf{E}(t)$ $=E \sin (\omega t) \mathbf{e}_{y}$, where $E$ and $\omega$ are the amplitude and frequency of the laser field. We use the following vector potentials for the static magnetic field $\mathbf{A}_{b}$ and the time-dependent laser field $\mathbf{A}_{e}(t)$ :

$$
\begin{gathered}
\mathbf{A}=\mathbf{A}_{b}+\mathbf{A}_{e}(t), \\
\mathbf{A}_{b}=B x \mathbf{e}_{y}, \\
\mathbf{A}_{e}(t)=A(t) \mathbf{e}_{y}=\frac{E c}{\omega} \cos (\omega t) \mathbf{e}_{y},
\end{gathered}
$$

where $B$ is the magnetic flux density. Then the Hamiltonian for the Schrödinger equation,

$$
i \hbar \frac{\partial}{\partial t}|\Psi(t)\rangle=H(t)|\Psi(t)\rangle
$$

is given as 


$$
H(t)=\frac{1}{2 m^{*}} \hat{p}_{x}^{2}+\frac{1}{2 m^{*}}\left(\hat{p}_{y}-\frac{e}{c} B \hat{x}-\frac{e}{c} A(t)\right)^{2}+V(\hat{x}, \hat{y}),
$$

where $m^{*}$ is the electron effective mass and $e$ is the electron charge. The last term in the Hamiltonian is an external potential with an explicit dependence on the spatial coordinates. Following Refs. 12 and 16, we define the unitary transformation

$$
|\Psi(t)\rangle=U(t)|\Phi(t)\rangle,
$$

with the unitary operator

$$
\begin{aligned}
U(t) \equiv & \exp \left(\frac{i}{\hbar} u_{1}(t)\right) \exp \left(\frac{i}{\hbar} u_{2}(t) \hat{x}\right) \exp \left(\frac{i}{\hbar} u_{3}(t) \hat{p}_{x}\right) \\
& \times \exp \left(\frac{i}{\hbar} u_{5}(t) \hat{p}_{y}\right) .
\end{aligned}
$$

Now we require that the Schrödinger equation for the state $|\Phi(t)\rangle$ does not contain the $A(t)$ term, i.e.,

$$
i \hbar \frac{\partial}{\partial t}|\Phi(t)\rangle=\left\{H_{0}+V\left[\hat{x}-u_{3}(t), \hat{y}-u_{5}(t)\right]\right\}|\Phi(t)\rangle
$$

with

$$
H_{0}=\frac{1}{2 m^{*}} \hat{p}_{x}^{2}+\frac{1}{2 m^{*}}\left(\hat{p}_{y}-\frac{e}{c} B \hat{x}\right)^{2} .
$$

This requirement leads to the following differential equations for the $u_{i}(t)$ 's:

$$
\begin{gathered}
-\dot{u}_{1}+\dot{u}_{2} u_{3}=\frac{1}{2 m^{*}}\left(\frac{e B}{c} u_{3}-\frac{e}{c} A(t)\right)^{2}+\frac{1}{2 m^{*}} u_{2}^{2}, \\
-\dot{u}_{2}=\frac{-e B}{m^{*}}\left(\frac{e B}{c} u_{3}-\frac{e}{c} A(t)\right), \\
-\dot{u}_{3}=\frac{1}{m^{*}} u_{2}, \\
-\dot{u}_{5}=\frac{1}{m^{*}}\left(\frac{e B}{c} u_{3}-\frac{e}{c} A(t)\right) .
\end{gathered}
$$

To solve these differential equations we impose the initial condition that

$$
u_{1}(0)=u_{2}(0)=u_{3}(0)=u_{5}(0)=0 .
$$

The solutions are

$$
\begin{aligned}
u_{1}(t)= & \frac{e^{2} E^{2}}{2 m^{*}}\left(\frac{1}{\omega_{c}^{2}-\omega^{2}}\right)^{2}\left[\frac{\omega_{c}^{3}}{2 \omega^{2}} \sin \left(2 \omega_{c} t\right)\right. \\
& +\frac{\omega}{4}\left(\frac{3 \omega_{c}^{2}}{\omega^{2}}-1\right) \sin (2 \omega t)+\left(\frac{\omega_{c}^{2}-\omega^{2}}{2}\right) t \\
& \left.-\frac{\omega_{c}^{3}}{\omega^{2}}\left\{\sin \left[\left(\omega_{c}-\omega\right) t\right]+\sin \left[\left(\omega_{c}+\omega\right) t\right]\right\}\right], \\
u_{2}(t)= & e E\left(\frac{\omega_{c}}{\omega_{c}^{2}-\omega^{2}}\right)\left[\frac{-\omega_{c}}{\omega} \sin \left(\omega_{c} t\right)+\sin (\omega t)\right], \\
u_{3}(t)= & \frac{e E}{m^{*}} \frac{\omega_{c}}{\omega}\left(\frac{1}{\omega_{c}^{2}-\omega^{2}}\right)\left[-\cos \left(\omega_{c} t\right)+\cos (\omega t)\right], \\
u_{5}(t)= & \frac{e E}{m^{*}}\left(\frac{1}{\omega_{c}^{2}-\omega^{2}}\right)\left[\frac{\omega_{c}}{\omega} \sin \left(\omega_{c} t\right)-\sin (\omega t)\right] .
\end{aligned}
$$

We note that the electron charge density is unchanged under these unitary transformation, i.e., $|\Psi|^{2}=|U \Phi|^{2}$.

\section{CHARGE FLUCTUATION}

The function $\Phi$ now satisfies Eq. (8). To solve this equation, we make use of the fact that $V$ is a weak perturbation even in the oscillatory system. Therefore, we solve the wave function $\Phi$ to the lowest order in $V$. The solutions for $V$ $=0$ are given by Laudau levels $\phi_{n k_{y}}$,

$$
\phi_{n k_{y}}(x, y, t)=\frac{1}{\sqrt{L}} e^{i k_{y} y} \xi_{n}\left(x-x_{0}\right) e^{-(i / \hbar) \epsilon_{n} t},
$$

where $L$ is the size of the sample, $x_{0}=k_{y} /(e B)=k_{y} l^{2}$ is the center coordinator, and $l$ is the magnetic length, $\epsilon_{n}=(n$ $+1 / 2) \hbar \omega_{c}\left(\omega_{c}=e B / m\right)$ is the cyclotron energy, and $\xi$ is the wave function for a linear harmonic oscillator.

We now employ this time-dependent wavefunction to calculate the electronic state in the potential $V$. The wave function of an electron under a local potential can be expanded using the Landau states

$$
\begin{aligned}
\Phi_{n k_{y}}(x, y, t)= & \sum_{n^{\prime} k_{y}^{\prime}}\left\{\delta_{n^{\prime} n} \delta_{k_{y}^{\prime} k_{y}}+a_{n^{\prime} k_{y}^{\prime}}(t)\right\} e^{i k_{y}^{\prime} y} \\
& \times \xi\left(x-x_{0}^{\prime}\right) e^{-(i / \hbar) \epsilon_{n^{\prime}} t} .
\end{aligned}
$$

The coefficient $a_{n k_{y}}(t)$ can be obtained using the timedependent perturbation method. Let us write the local potential as

$$
\begin{aligned}
V[x & \left.-u_{3}(t), y-u_{5}(t)\right] \\
& =\int d \mathbf{q}^{\prime} e^{i q_{x}^{\prime}\left[x-u_{3}(t)\right]} e^{i q_{y}^{\prime}\left(y-u_{5}(t)\right)} V\left(\mathbf{q}^{\prime}\right) .
\end{aligned}
$$


Making using of the generating function of the Bessel function,

$$
e^{i \alpha \sin x}=\sum_{m} J_{m}(\alpha) e^{i m x}, \quad e^{i \alpha \cos x}=\sum_{m} i^{m} J_{m}(\alpha) e^{i m x},
$$

where $J_{m}$ is the Bessel function of the first kind, the potential can be written as

$$
\begin{aligned}
& V\left[x-u_{3}(t), y-u_{5}(t)\right] \\
& =\int d \mathbf{q} e^{i q_{x} x} e^{i q_{y} y} V(\mathbf{q}) \sum_{m_{1}, m_{2}} i^{m_{1}+m_{2}} J_{m_{1}}\left(q_{x} \gamma_{b} \omega_{c} / \omega\right) \\
& \quad \times J_{m_{2}}\left(-q_{x} \gamma_{b} \omega_{c} / \omega\right) e^{i\left(m_{1} \omega_{c}+m_{2} \omega\right) t} \\
& \quad \times \sum_{m_{3}, m_{4}} J_{m_{3}}\left(-q_{y} \gamma_{b} \omega_{c} / \omega\right) J_{m_{4}}\left(q_{y} \gamma_{b}\right) e^{i\left(m_{3} \omega_{c}+m_{4} \omega\right) t},
\end{aligned}
$$

where $\gamma_{b}=e E /\left[m^{*}\left(\omega_{c}^{2}-\omega^{2}\right)\right]$. We assume that the local potential is weak and use the time-dependent perturbation to calculate the change of electronic state. The first order correction can be written as

$$
\begin{aligned}
& a_{n^{\prime} k_{y}^{\prime}}(t)=\frac{1}{i \hbar} \int d \mathbf{q} V(\mathbf{q}) \sum_{m_{1}, m_{2}} i^{m_{1}+m_{2}} J_{m_{1}}\left(q_{x} \gamma_{b} \omega_{c} / \omega\right) \\
& \times J_{m_{2}}\left(-q_{x} \gamma_{b} \omega_{c} / \omega\right) \sum_{m_{3}, m_{4}} \\
& \times J_{m_{3}}\left(-q_{y} \gamma_{b} \omega_{c} / \omega\right) J_{m_{4}}\left(q_{y} \gamma_{b}\right) \\
& \times \int_{-\infty}^{t} d t^{\prime} e^{(i / \hbar)\left(\epsilon_{n^{\prime}}-\epsilon_{n}\right) t^{\prime}} e^{i\left(m_{1} \omega_{c}+m_{2} \omega\right) t^{\prime}} \\
& \times e^{i\left(m_{3} \omega_{c}+m_{4} \omega\right) t^{\prime}} \frac{1}{L} \\
& \times \int d y e^{-i\left(k_{y}^{\prime}-k_{y}-q_{y}\right) y} M_{n^{\prime} n}\left(q_{x}, k_{y}^{\prime}, k_{y}\right) .
\end{aligned}
$$

The integration over $x$ and $y$ yields

$$
\begin{aligned}
& M_{n^{\prime} n}\left(q_{x}, k_{y}^{\prime}, k_{y}\right) \\
& =\int d x \xi_{n^{\prime}}\left(x-x_{0}^{\prime}\right) e^{i q_{x} x} \xi_{n}\left(x-x_{0}\right) \\
& =\exp \left(-\frac{l^{2} q^{2}}{2}\right) \exp \left(i l^{2} k_{y} q_{x}\right) \exp \left(\frac{i}{2} l^{2} q_{x} q_{y}\right)\left(\frac{2^{n} n_{<} !}{2^{n_{>}} n_{>} !}\right)^{\frac{1}{2}} \\
& \quad \times l^{n>-n_{<}}\left(\theta q_{y}+i q_{x}\right)^{n_{>}-n_{<}} L_{n_{<}}^{n_{>}-n_{<}}\left(\frac{l^{2} q^{2}}{2}\right) .
\end{aligned}
$$

Here $n_{>}\left(n_{<}\right)$is the larger (smaller) of $\left(n, n^{\prime}\right)$. Combining these results, we obtain

$$
\begin{aligned}
a_{n^{\prime} k_{y}^{\prime}}(t)= & -\int d \mathbf{q} V(\mathbf{q}) \delta_{k_{y}^{\prime}, k_{y}+q_{y}} \sum_{\{m\}} I\left(\{m\}, q_{x}, q_{y}\right) \\
& \times D_{n^{\prime} n}(\{m\}) E(\{m\}, t) \times e^{(i / \hbar)\left(\epsilon_{n^{\prime}}-\epsilon_{n}\right) t} \\
& \times M_{n^{\prime} n}\left(q_{x}, k_{y}+q_{y}, k_{y}\right),
\end{aligned}
$$

where we have defined

$$
\begin{gathered}
\sum_{\{m\}}=\sum_{m_{1}} \sum_{m_{2}} \sum_{m_{3}} \sum_{m_{4}} \\
I\left(\{m\}, q_{x}, q_{y}\right)=i^{m_{1}+m_{2}} J_{m_{1}}\left(\frac{q_{x} \gamma_{b} \omega_{c}}{\omega}\right) J_{m_{2}}\left(\frac{-q_{x} \gamma_{b} \omega_{c}}{\omega}\right) \\
\times J_{m_{3}}\left(\frac{-q_{y} \gamma_{b} \omega_{c}}{\omega}\right) J_{m_{4}}\left(q_{y} \gamma_{b}\right)
\end{gathered}
$$

$$
E(\{m\}, t)=e^{i\left(m_{1}+m_{3}\right) \omega_{c} t+i\left(m_{2}+m_{4}\right) \omega t}
$$

and

$$
D_{n^{\prime} n}(\{m\})=\frac{1}{\epsilon_{n^{\prime}}-\epsilon_{n}+\left(m_{1}+m_{3}\right) \hbar \omega_{c}+\left(m_{2}+m_{4}\right) \hbar \omega} .
$$

Now the wave function up to the first order is given as

$$
\begin{aligned}
\Phi_{n k_{y}}(\mathbf{r}, t)= & \phi_{n k_{y}}(\mathbf{r}, t)+\sum_{n^{\prime} k_{y}^{\prime}} a_{n^{\prime} k_{y}^{\prime}}(t) \phi_{n^{\prime} k_{y}^{\prime}}(\mathbf{r}, t) \\
= & \frac{1}{\sqrt{L}} e^{i k_{y} y} e^{-\frac{i}{\hbar} \epsilon_{n} t}\left[\xi_{n}\left(x-l^{2} k_{y}\right)-\int d \mathbf{q} V(\mathbf{q}) \sum_{\{m\}}\right. \\
& \times I\left(\{m\}, q_{x}, q_{y}\right) \times E(\{m\}, t) e^{i q_{y} y} \xi_{n}\left(x-x_{0}^{\prime}\right) \\
& \left.\times \sum_{n^{\prime}} M_{n^{\prime} n}\left(q_{x}, k_{y}+q_{y}, k_{y}\right) D_{n^{\prime} n}(\{m\})\right],
\end{aligned}
$$

where $x_{0}^{\prime}=\left(k_{y}+q_{y}\right)^{2}$. The fluctuation of charge distribution (induced charge density) can now be calculated:

$$
\begin{aligned}
\rho_{n k_{y}}(\mathbf{r}, t)= & -e\left\{\left[U \Phi_{n k_{y}}(\mathbf{r}, t)\right]^{*} U \Phi_{n k_{y}}(\mathbf{r}, t)\right. \\
& \left.-\left[U \phi_{n k_{y}}(\mathbf{r}, t)\right]^{*} U \phi_{n k_{y}}(\mathbf{r}, t)\right\} .
\end{aligned}
$$

Neglecting high order terms in $V$, we obtain 


$$
\begin{aligned}
\rho_{n k_{y}}(\mathbf{r}, t)= & \frac{-e}{L} \xi_{n}\left[x-x_{0}-u_{3}(t)\right] \int d \mathbf{q} V(\mathbf{q}) \\
& \times \sum_{\{m\}} I\left(\{m\}, q_{x}, q_{y}\right) \sum_{n^{\prime}} D_{n^{\prime} n}(\{m\}) \\
& \times \xi_{n^{\prime}}\left[x-x_{0}^{\prime}-u_{3}(t)\right]\left[M_{n^{\prime} n}\left(q_{x}, k_{y}+q_{y}, k_{y}\right)\right. \\
& \times E(\{m\}, t) e^{i q_{y}\left[y-u_{5}(t)\right]}+(-)^{m_{1}+m_{2}} M_{n^{\prime} n}^{*} \\
& \left.\times\left(q_{x}, k_{y}+q_{y}, k_{y}\right) E^{*}(\{m\}, t) e^{-i q_{y}\left[y-u_{5}(t)\right]}\right] .
\end{aligned}
$$

We now take the Fourier transformation, $\rho_{n k_{y}}(\mathbf{q}, t)$ $=\int d \mathbf{r} e^{-i \mathbf{q} \cdot \mathbf{r}} \rho_{n k_{y}}(\mathbf{r}, t)$,

$$
\begin{aligned}
\rho_{n k_{y}}(\mathbf{q}, t)= & \frac{-e}{L} \int d \mathbf{q}^{\prime} V\left(\mathbf{q}^{\prime}\right) \sum_{\{m\}} I\left(\{m\}, \mathbf{q}^{\prime}\right) \sum_{n^{\prime}} D_{n^{\prime} n}(\{m\}) \\
& \times e^{-i q_{x}^{\prime} u_{3}(t)}\left[M_{n^{\prime} n}\left(-q_{x}^{\prime}, k_{y}+q_{y}, k_{y}\right) M_{n^{\prime} n}\right. \\
& \times\left(q_{x}, k_{y}+q_{y}, k_{y}\right) E(\{m\}, t) e^{-i q_{y} u_{5}(t)} \delta_{q_{y}^{\prime}, q_{y}} \\
& +(-)^{m_{1}+m_{2}} M_{n^{\prime} n}\left(-q_{x}^{\prime}, k_{y}+q_{y}, k_{y}\right) M_{n^{\prime} n}^{*} \\
& \left.\times\left(q_{x}, k_{y}+q_{y}, k_{y}\right) E^{*}(\{m\}, t) e^{i q_{y} u_{5}(t)} \delta_{q_{y}^{\prime},-q_{y}}\right] .
\end{aligned}
$$

Integrating over $k_{y}$ results in a delta function $\delta\left(q_{x}-q_{x}^{\prime}\right)$ for the first term in the square bracket and $\delta\left(q_{x}+q_{x}^{\prime}\right)$ for the second term. Now for the second term in the square bracket, we change $m_{i}$ to $-m_{i}(i=1,2,3,4)$ and make use of the fact, $J_{-m}(x)=(-1)^{m} J_{m}(x)$. We obtain the following result for the density fluctuation:

$$
\begin{aligned}
\rho_{n}(\mathbf{q}, t)= & \frac{-2 \pi e}{l^{2}} V(\mathbf{q}) e^{-i q_{x} u_{3}(t)} e^{-i q_{y} u_{5}(t)} \\
& \times \sum_{n^{\prime}} C_{n n^{\prime}}(t) \sum_{\{m\}} I(\{m\}, \mathbf{q}) E(\{m\}, t) \\
& \times\left[\frac{1}{\epsilon_{n^{\prime}}-\epsilon_{n}+\left(m_{1}+m_{3}\right) \hbar \omega_{c}+\left(m_{2}+m_{4}\right) \hbar \omega}\right. \\
& \left.+\frac{1}{\epsilon_{n^{\prime}}-\epsilon_{n}-\left(m_{1}+m_{3}\right) \hbar \omega_{c}-\left(m_{2}+m_{4}\right) \hbar \omega}\right],
\end{aligned}
$$

where

$$
C_{n n^{\prime}}(q)=\frac{n_{<}}{n_{>}} \exp (-Y) Y^{\left(n_{>}-n_{<}\right)}\left[L_{n_{<}}^{n_{>}-n_{<}}(Y)\right]^{2},
$$

with $Y=(1 / 2)(q l)^{2}$.

Now the total density fluctuation of the system is given as

$$
\rho(\mathbf{q}, t)=\sum_{n} f_{n} \rho_{n}(\mathbf{q}, t)
$$

where $f_{n}=\left[\exp \left(\left(\epsilon_{n}-\mu-E_{\gamma}\right) / k_{B} T\right)+1\right]^{-1}$ is the Fermi distribution function. Here $E_{\gamma}=2 \gamma_{b} \omega$ is the energy of the laser field, $\mu$ is the chemical potential and $k_{B}$ is the Boltzmann constant. Substituting Eq. (32) into Eq. (33),

$$
\begin{aligned}
\rho(\mathbf{q}, t)= & -e V(\mathbf{q}) e^{-i q_{x} u_{3}(t)} e^{-i q_{y} u_{5}(t)} \\
& \times \sum_{\{m\}} I(\{m\}, \mathbf{q}) E(\{m\}, t) \Pi(q,\{m\}),
\end{aligned}
$$

where $\Pi(q,\{m\})$ is defined as

$$
\begin{aligned}
\Pi(q,\{m\})= & \frac{2 \pi}{l^{2}} \sum_{n n^{\prime}} C_{n n^{\prime}}(q) \\
& \times \frac{f_{n}-f_{n^{\prime}}}{\epsilon_{n^{\prime}}-\epsilon_{n}+\left(m_{1}+m_{3}\right) \hbar \omega_{c}+\left(m_{2}+m_{4}\right) \hbar \omega} .
\end{aligned}
$$

If the electric field $\mathrm{E}=0$, only $J_{0}$ can survive. In this case we recover the known result

$$
\rho(\mathbf{q})=-e V(\mathbf{q}) \Pi(q, 0) .
$$

\section{DISCUSSIONS}

It can be seen that the induced charge density oscillates in time with two characteristic frequencies, the frequency of the THz laser, $\omega$, and the the cyclotron frequency $\omega_{c}$. This behavior is rather interesting. In the absence of the laser field, the induced density in a magnetically quantized system due to a static potential $V$ is not time dependent, i.e., it is not oscillating in time with the cyclotron frequency. Now we apply a time-dependent laser field. It is expected that all physical quantities of the system will oscillate with the laser frequency and it higher multiples. What is interesting here is that the induced density is oscillating with a frequency which is the sum of the multiple of the laser frequency and the multiple of the cyclotron frequency. When the magnetic approaches zero, the oscillation in the electronic states and the induced charge density is dominated by the laser frequency. On the other hand, if the magnetic field is high such that $\omega_{c} \gg \omega$, the electronic states and the induced charge density oscillate very rapidly with the frequency $\omega_{c}$. Therefore, even a slow varying time-dependent electrical field and can lead to a rapid oscillatory behavior in electronic properties with a frequency corresponding to the natural energy gap of the system, $\left(\omega_{c}\right.$ in the present case). Such oscillation can only survive if the intensity of the laser field is strong. It should be noted that while there is a rapid oscillation with frequency $\omega_{c}$ in the limit of $\omega \ll \omega_{c}$, there is also a slow oscillation with frequency $\omega$. Furthermore, as $\omega$ decreases, the arguments of all Bessel functions increase rapidly and thus the amplitude of the oscillation also decreases very rapidly as 
$\omega \rightarrow 0$. In the limit of $\omega=0$, the amplitudes of all oscillations go to zero regardless of the oscillating frequencies. In this case the density fluctuation of the system becomes time independent.

The density fluctuation also oscillates with the electric field at fixed magnetic field and laser frequency. This oscillation is due to the oscillatory behavior of various Bessel functions. We notice that the argument of the Bessel function can be written as $q_{y} \gamma_{b} \omega_{c} / \omega=(e E l / \hbar \omega)\left(q_{y} l\right) /\left(1-\omega^{2} / \omega_{c}^{2}\right)$. The parameter $r=e E l / \hbar \omega$ is the ratio of the energy gained by an electron in the laser field over the distance equivalent to the magnet length to the energy of the photon. For small $r$ or high $\omega$, the dominant contribution is from $J_{0}$. In this limit, the density fluctuation is approximately given as $\rho(E)-\rho(E=0) \propto r^{2}$. For large $r$ or small $\omega_{c}^{2}-\omega^{2}$, $J_{m}\left(\alpha \gamma_{b}\right) \sim \sqrt{2 / \pi \alpha \gamma_{b}} \cos \left(\alpha \gamma_{b}-m \pi / 2+\pi / 4\right)$. While the density fluctuation oscillates with the electric field, the amplitude goes to zero as the electric field increases. This is a rather interesting result which indicates that as the electrical field increases, the induce charge density decreases. This reduction of the induced charge density under a strong electrical field may have the some origin as the experimentally observed suppression of the dc conductivity under an intense laser. ${ }^{4,7}$ Finally we comment on the difference between the case of strong field and the case of near resonance $(\omega$ $\left.\approx \omega_{c}\right)$. While both cases can cause an increased $\gamma_{b}$, these two are fundamentally different. A strong $E$ directly causes a strong electron-photon interaction. The situation of $\omega \rightarrow \omega_{c}$ represents an electron-photon resonant state. The quantities that affect the density response are $u_{3}$ and $u_{5}$. At resonance, $u_{3}$ is linear in $t$ and $u_{5}$ is quadratic in $t$. This will lead to a quite different time dependence of the induced charge density.

\section{ACKNOWLEDGMENTS}

This work was supported by the Japan Society for Promotion of Science and the Australian Academy of Sciences through the Australia-Japan Bilateral Scientific Collaboration Program. J. C. Cao acknowledges support by the Key Project of the National Natural Science Foundation of China, the Special Funds for Major State Basic Research Project (2001CCA02800G and 20000683), and the Special Funds for Shanghai Optic Engineering (011661075).
${ }^{1}$ R. Köhler et al., Nature (London) 417, 156 (2002).

${ }^{2}$ M. A. Odnoblyudov, I. N. Yassievich, M. S. Kogan, Yu. M. Gelperin, and K. A. Chao, Phys. Rev. Lett. 83, 644 (1999).

${ }^{3}$ Yu. P. Gousev, I. V. Altukhov, K. A. Korolev, V. P. Sinis, M. S. Kogan, E. E. Haller, M. A. Odnoblyudov, I. N. Yassievich, and K. A. Chao, Appl. Phys. Lett. 75, 757 (1999).

${ }^{4}$ N. G. Asmar, A. G. Markelz, E. G. Gwinn, J. Cerne, M. S. Sherwin, K. L. Campman, P. E. Hopkins, and A. C. Gossard, Phys. Rev. B 51, 18041 (1995).

${ }^{5}$ W. Xu and C. Zhang, Appl. Phys. Lett. 68, 3305 (1996).

${ }^{6}$ W. Xu and C. Zhang, Phys. Rev. B 55, 5259 (1997).

${ }^{7}$ N. G. Asmar, J. Cerne, A. G. Markelz, E. G. Gwinn, M. S. Sherwin, K. L. Campman, and A. C. Gossard, Appl. Phys. Lett. 68, 829 (1996).

${ }^{8}$ A. G. Markelz, N. G. Asmar, B. Brar, and E. G. Gwin, Appl. Phys. Lett. 69, 3975 (1996).
${ }^{9}$ B. N. Murdin, W. Heiss, G. J. G. M. Langerak, S.-C. Lee, I. Galbraith, G. Strasser, E. Gornik, M. Helm, and C. R. Pidgeon, Phys. Rev. B 55, 5171 (1997).

${ }^{10}$ C. J. G. M. Langerak, B. N. Murdin, B. E. Cole, J. M. Chamberlain, M. Henini, M. Pate, and G. Hill, Appl. Phys. Lett. 67, 3453 (1995).

${ }^{11}$ T. A. Vaughan, R. J. Nicholas, C. J. G. M. Langerak, B. N. Murdin, C. R. Pidgeon, N. J. Mason, and P. J. Walker, Phys. Rev. B 53, 16481 (1996).

${ }^{12}$ C. Zhang, Appl. Phys. Lett. 78, 4187 (2001).

${ }^{13}$ P. Orellana, F. Claro, and E. Anda, Phys. Rev. B 62, 9959 (2000).

14 P. Orellana and F. Claro, Appl. Phys. Lett. 75, 1643 (1999).

${ }^{15}$ C. Zhang and W. Xu, Physica B 298, 333 (2001).

${ }^{16}$ C. Zhang, Phys. Rev. B 65, 153107 (2002). 